\title{
MORTALIDAD EN CERATITIS CAPITATA (WIEDEMANN) (DIPTERA: TEPHRITIDAE) POR DIVERSAS CEPAS DE BEAUVERIA BASSIANA (BALS.) VUILLEMIN, EN CONDICIONES DE LABORATORIO
}

\author{
José A. Muñoz, William de LA Rosa $(\dagger)$ y Jorge TOLEDO \\ Departamento de Entomología Tropical. El Colegio de la Frontera Sur. Apartado Postal 36. \\ Tapachula, Chiapas.30700. MÉXICO. jtoledo@ecosur.mx
}

Muñoz, J. A., W. de la Rosa y J. Toledo. 2009. Mortalidad en Ceratitis capitata (Wiedemann) (Diptera: Tephritidae) por Diversas Cepas de Beauveria bassiana (Bals.) Vuillemin, en Condiciones de Laboratorio. Acta Zoológica Mexicana (n. s.), 25(3): 609-624.

RESUMEN. Bajo condiciones de laboratorio $\left(27 \pm 2{ }^{\circ} \mathrm{C}\right.$ de temperatura, $80 \pm 5 \%$ de H. R. y $12: 12$ horas luz: obscuridad), se caracterizaron mediante bioensayos por inmersión, la patogenicidad de 16 cepas del hongo Beauveria bassiana (Bals.) Vuillemin aisladas de diferentes hospederos sobre hembras de Ceratitis capitata (Wiedemann). El rango de mortalidad que se observó fue de 9.1 a $91.9 \%$; el tiempo letal medio de la cepa más virulenta fue de $\left(\mathrm{TL}_{50}=3.83\right.$ días $)$, mientras que en la cepa con menor virulencia, el tiempo letal medio fue de $\left(\mathrm{TL}_{50}=17.64\right.$ días). En una segunda fase, las cepas que registraron mayor actividad biológica fueron evaluadas mediante el mismo procedimiento, en donde se registraron elevados índices de mortalidad, producción y recuperación de conidios a partir de los insectos infectados. De acuerdo con estos resultados, se discute el potencial que cinco cepas de $B$. bassiana tienen para ser evaluadas en condiciones de campo y determinar si pueden ser utilizadas en el control de dicha plaga.

Palabras clave: Hongos entomopatógenos, B. bassiana, control microbiano, mosca del mediterráneo.

Muñoz, J. A., W. de la Rosa \& J. Toledo. 2009. Ceratitis capitata (Wiedemann) (Diptera: Tephritidae) mortality by different Beauveria bassiana (Bals.) Vuillemin strains under laboratory conditions. Acta Zoológica Mexicana (n. s.), 25(3): 609-624.

ABSTRACT. Under laboratory conditions of $27 \pm 2{ }^{\circ} \mathrm{C}, 80 \pm 5 \% \mathrm{R}$. H. and 12:12 L:D, we performed a characterization of 16 strains of the entomopathogenic fungus Beauveria bassiana (Bals.) Vuillemin, through bioessays by immersion. The strains were isolated from different hosts and their pathogenicity was evaluated on Ceratitis capitata (Wiedemann) females. Results showed that mortality of flies ranged from 9.1 to $91.9 \%$. Average lethal time $\left(\mathrm{LT}_{50}\right)$ of the most pathogenic strain was 3.8 days; while that the less pathogenic strain took 17.6 days. In a second series of experiments, the strains that registered the highest biological activity were evaluated as mentioned above. These strains showed high levels of mortality, spore production and spore recovery from the infected insects. According with these results, we discuss the potential that five strains of $B$. bassiana have be tested under field conditions to determinate if can be used in the control of this pest.

Key words: Entomopathogenic fungi, B. bassiana, microbial control, Mediterranean fruit fly.

Recibido: 10/03/2009; aceptado: 10/08/2009. 


\section{INTRODUCCIÓN}

La mosca del Mediterráneo (Ceratitis capitata Wied.) (Diptera: Tephritidae), es una plaga de origen Africano que se ha extendido más allá de su área de origen y ha colonizado importantes áreas tropicales y subtropicales en diversas partes del mundo (Gutiérrez 1976). Los países que actualmente no están infestados por dicha plaga, como es el caso de México, evitan a toda costa que se introduzca y se establezca en sus territorio (Villaseñor et al. 2000, Hendrichs et al. 2002). De establecerse en territorio mexicano sería motivo de cumplir con las rigurosas medidas fitosanitarias que se establecerían para permitir la movilización y comercialización de frutas, que en muchas ocasiones generan mayores pérdidas que la misma plaga (Gutiérrez 1976, Enkerlin et al. 1989).

Los programas de manejo que se llevan a cabo en los países con presencia de esta plaga, utilizan varias tácticas de control incluyendo la aplicación de insecticidas químicos convencionales dirigidos al suelo o asperjados al follaje, bajo un enfoque integral (Saul et al. 1983, Penrose 1993, Villaseñor et al. 2000), destacando la Técnica del Insecto Estéril (TIE) como elemento clave (Villaseñor et al. 2000, Hendrichs et al. 2002). Sin embargo, es necesario desarrollar otras alternativas que puedan considerarse complementarias, haciendo énfasis en aquellas que tengan un menor impacto en el ambiente y mayor aceptación por productores de frutas y café en sistemas agrícolas orgánicos. De esta manera, como nuevas alternativas se ha incorporado el uso del spinosad (Success 0.02 CB o GF-120), estaciones cebo y trampeo masivo (Vargas et al. 2001, Zaragoza \& Fernández. 2002). Por lo que se ha considerado que los hongos entomopatógenos ofrecen un gran potencial, ya que está comprobado su eficacia como agentes de control de otras plagas agrícolas (Lecuona et al. 1996), y en el caso de moscas de la fruta se ha demostrado que algunas cepas provocan altas mortalidades en adultos (Castillo et al. 2000, De la Rosa et al. 2002).

La fase conidial (esporas) de varias cepas de los hongos entomopatógenos Beauveria bassiana (Bals.) Vuillemin y Metharizuim anisopliae (Metsch.) Sorokin, de diferentes regiones geográficas, se han evaluado en condiciones de laboratorio contra las diferentes fases biológicas de varias especies de moscas de la fruta (García et al. 1984, Espin et al. 1989, Castillo et al. 2000, De la Rosa et al. 2002, Ekesi et al. 2002, Yee \& Lacey 2005). Por lo tanto, se requiere determinar la cantidad de conidios por concentración a evaluarse, tanto en los bioensayos como en los experimentos de campo. A nivel de laboratorio, el método más utilizado es por inmersión de las fases biológicas del insecto (larva, pupa o adulto) en solución de conidios (De la Rosa $e t$ al. 2002), y en condiciones de campo es necesario desarrollar dispositivos de infección o de transmisión del inoculo (Dimbi et al. 2003), incluyendo las aplicaciones dirigidas al suelo y otras formas en que los conidios hagan contacto con los adultos de la plaga (Lezama-Gutiérrez et al. 2000, Ekesi et al. 2005, Toledo et al. 2007). 
Cabe mencionar que no todas las cepas de los hongos entomopatógenos son efectivas para causar la infección de larvas y pupas o pueden ocasionar altas mortalidades en adultos de moscas de la fruta (De la Rosa et al. 2002), por lo que es necesario caracterizar su virulencia mediante bioensayos. De esta forma, las cepas procedentes de una región geográfica, caracterizadas a través de dicha técnica y se comprueba que poseen una alta actividad biológica tendrán mayor potencial, por lo que deben evaluarse en los programas de manejo de moscas de la fruta en la misma región.

El objetivo de este trabajo fue caracterizar mediante bioensayos, diversas cepas del hongo B. bassiana contra adultos de la mosca del mediterráneo y seleccionar aquellas con potencial para integrarlas a las acciones que se realizan en los programas de manejo de dicha plaga, en sistemas agrícolas convencionales y principalmente en agroecosistemas orgánicos.

\section{MATERIALES Y MÉTODOS}

El estudio se realizó en el laboratorio de Patología de Insectos de la Línea Manejo Integrado de Plagas, en colaboración con la Línea Ecología de Poblaciones de Moscas de la Fruta, pertenecientes a El Colegio de la Frontera Sur (ECOSUR), Unidad Tapachula, en Tapachula, Chiapas, México.

Material biológico. Se evaluaron 16 cepas del hongo B. bassiana aisladas a partir de diferentes hospederos (Cuadro 1). Para la producción del inóculo se utilizó el medio de cultivo Agar Dextrosa Saboraud (ADS) (65 g de agar + 1 litro de agua destilada y $5 \mathrm{~g}$ de extracto de levadura). El medio de cultivo se esterilizó a $120^{\circ} \mathrm{C}$ por 15 minutos $\left(15 \mathrm{lb} / \mathrm{pg}^{2}\right)$ y después fue vertido en tubos de ensayos y en cajas de Petri estériles, las cuales se dejaron en reposo por tres días para que gelificara el medio de cultivo. La siembra de los conidios en los tubos de ensayos y en las cajas de Petri se hizo con un asa bacteriológica estéril en el interior de una campana de flujo laminar mediante la técnica de estrías. Posteriormente el material biológico se incubo por 15 días a una temperatura de $27 \pm 2^{\circ} \mathrm{C}$, humedad relativa de $80 \pm 5 \%$ y un fotoperíodo de 12:12 L:O. Los conidios fueron colectados raspando con un asa bacteriológica la superficie del medio de cultivo y después fueron depositados en contenedores de plástico color ámbar, los cuales se almacenaron a $4{ }^{\circ} \mathrm{C}$ hasta su eventual utilización en los bioensayos.

Los adultos de $C$. capitata, fueron proporcionados en estado de pupa (dos días antes de la emergencia de adultos, previamente irradiada con Cobalto 60 a una dosis de 145 Gy) por el Laboratorio de Cría y Esterilización del Programa Moscamed, ubicado en Metapa de Domínguez, Chiapas, México, en donde se desarrollaron en una dieta larvaria de acuerdo con las especificaciones descritas por DomínguezGordillo et al. (2000). Las pupas fueron colocadas en jaulas de vidrio de $27 \mathrm{dm}^{3} \mathrm{y}$ una vez que emergieron los adultos, fueron separados por sexos y alimentados con 
una mezcla de proteína hidrolizada + azúcar (relación, 1:3) y el agua se les suministró en viales cubiertos con torundas de algodón. Las jaulas con los insectos permanecieron en el laboratorio a una temperatura de $26 \pm 1{ }^{\circ} \mathrm{C}$, humedad relativa de $75 \pm 5 \%$ y un fotoperíodo de 12:12 horas de luz-oscuridad.

Antes de realizar los bioensayos se determinó la viabilidad de los conidios de cada cepa utilizando la técnica de microcultivos descrita por Jiménez (1992). La viabilidad se determinó en base a la presencia del tubo germinativo del hongo y se consideró una cepa viable para realizar los bioensayos cuando presentaron $>90 \%$ de conidios germinados.

Cuadro 1. Hospederos originales de donde fueron aisladas las cepas de Beauveria bassiana, evaluadas contra hembras de la mosca del Mediterráneo.

\begin{tabular}{lll}
\hline Cepa & Hospedero & Orden: Familia \\
\hline $\mathrm{Bb} 2$ & Hypotenemus hampei & Coleoptera: Scolytidae \\
$\mathrm{Bb} 4$ & Hypotenemus hampei & Coleoptera: Scolytidae \\
$\mathrm{Bb} 6$ & Hypotenemus hampei & Coleoptera: Scolytidae \\
$\mathrm{Bb} 7$ & Atta mexicana & Hymenoptera: Formicidae \\
$\mathrm{Bb} 8$ & Leptinotarsa sp. & Coleoptera: Crysomelidae \\
$\mathrm{Bb} 9$ & Ligus sp. & Hemiptera: Miridae \\
$\mathrm{Bb} 12$ & Nezara viridula & Hemiptera: Pentatomidae \\
$\mathrm{Bb} 13$ & Boverin & Producto comercial \\
$\mathrm{Bb} 15$ & Chalcodermus aeneus & Coleoptera: Curculionidae \\
$\mathrm{Bb} 16$ & Chalcodermus aeneus & Coleoptera: Curculionidae \\
$\mathrm{Bb} 17$ & No determinado & Lepidoptera \\
$\mathrm{Bb} 18$ & No determinado & Lepidoptera \\
$\mathrm{Bb} 19$ & No determinado & Lepidoptera \\
$\mathrm{Bb} 25$ & Hypotenemus hampei & Coleoptera: Scolytidae \\
$\mathrm{Bb} 26$ & Hypotenemus hampei & Coleoptera: Scolytidae \\
$\mathrm{Bb} 27$ & No determinado & Coleoptera: Scarabeidae \\
\hline
\end{tabular}

Patogenicidad hacia hembras. En todas las pruebas se utilizaron solamente hembras debido a que estudios previos indicaron que tienen mayor tolerancia a la infección del hongo B. bassiana. Todas las cepas de $B$. bassiana fueron preparadas a partir de $1 \mathrm{~g}$ de conidios diluidos en $100 \mathrm{~mL}$ de agua destilada estéril $+0.025 \%$ de glicerina que se utilizó como dispersante-adherente y se estandarizaron a una concentración de 1 x $10^{8}$ conidios/mL calculado mediante conteos realizados con un hemocitómetro. 
En esta prueba se utilizaron 250 hembras de $C$. capitata, colocándolas en grupos de 50 individuos/tubo de ensayo, después se adicionaron $5 \mathrm{~mL}$ de solución fungosa, agitándolo suavemente para que las moscas se impregnaran con la solución. Posteriormente las moscas fueron transferidas a cajas de Petri y con papel absorbente se eliminó el exceso de humedad. En cada unidad experimental (contenedores de plástico de 1 litro de capacidad), se colocaron 10 hembras, para tener cinco repeticiones por cepa, realizando el experimento cinco veces. Para evitar que las mocas murieran por inanición, cada contenedor fue provisto de alimento a base de proteína hidrolizada enzimáticamente + azúcar (1:3) y el agua fue suministrada en viales cubiertos con torundas de algodón.

Las unidades experimentales se colocaron en un laboratorio bajo condiciones controladas de temperatura de $27 \pm 2{ }^{\circ} \mathrm{C}$, humedad relativa de $80 \pm 5 \%$ y un fotoperíodo de 12:12 horas luz-oscuridad en donde permanecieron por 10 días. Durante este tiempo fueron revisadas cada $24 \mathrm{~h}$, para registrar el número de moscas muertas. Las moscas muertas fueron colocadas en cámara húmeda para promover el crecimiento de micelios y confirmar que la muerte fue por infección del hongo.

Tiempo letal medio $\left(\mathbf{T L}_{\mathbf{5 0}}\right)$. Para estimar el tiempo letal medio $\left(\mathrm{TL}_{50}\right)$, se preparó una solución fungosa por cepa de acuerdo a la metodología que fue descrita para los bioensayos de patogenicidad. Posteriormente 50 hembras fueron tratadas con la solución de cada cepa de B. bassiana, realizando cinco repeticiones por cada una, 250 hembras en total. Todas las unidades experimentales permanecieron bajo las condiciones controladas antes indicadas y llevando un registro diario por concentración, cepa de hongo y fecha de los insectos muertos, los cuales fueron colocados en cámaras húmedas para estimular el crecimiento micelial y verificar que la muerte ocurrió por acción del hongo, tomando en cuenta la presencia del micelio.

Concentración letal media $\left(\mathbf{C L}_{\mathbf{5 0}}\right)$. De acuerdo con los resultados de la prueba de patogenicidad, se seleccionaron las cinco cepas más virulentas para las hembras de la mosca del Mediterráneo. Las dosis intermedias de la solución fungosa se prepararon a las concentraciones de 1.0, 0.6, 0.3, 0.1, 0.06, 0.03, 0.01, 0.006, 0.003 y $0.001 \%$. Por cada concentración se manejó un testigo (tratado solamente con agua) de 50 insectos, con al menos tres repeticiones (150 individuos en total). Las concentraciones evaluadas fueron calculadas en base a los resultados obtenidos en la prueba de la ventana biológica mediante el análisis Probit. La solución de conidios de cada cepa se preparó de acuerdo al procedimiento descrito en la prueba de patogenicidad y las unidades experimentales se colocaron en un cuarto de cría con condiciones ambientales controladas, una temperatura de $27 \pm 2{ }^{\circ} \mathrm{C}$, humedad relativa de $80 \pm 5 \%$ y fotoperíodo de 12:12 horas luz-oscuridad.

Esporulación de $B$. bassiana en hembras infectadas. Las moscas muertas fueron colocadas en cámara húmeda para estimular la emergencia del micelio, confirmándose mediante observaciones realizadas utilizando un microscopio 
estereoscópico. Diariamente se llevó un registro de las moscas infectadas por concentración del inoculo aplicado y por cepa, actividad que se realizó de manera consecutiva hasta que murieron todos los insectos tratados. La esporulación fue calculada como el porcentaje de hembras muertas que presentaron los signos característicos de infección de B. bassiana, cinco o seis días después de haber sido colocadas en la cámara húmeda. Este estudio tuvo una distribución completamente al azar en donde cada cepa fue analizada considerando a cada concentración como tratamiento y por cada tratamiento hubo tres repeticiones, dando un total de 30 unidades experimentales. Además en un segundo diseño experimental, se realizó un análisis considerando a las diferentes cepas como tratamientos y las repeticiones fueron entre las mismas concentraciones, en este caso hubo un total de 15 unidades experimentales.

Recuperación de conidios de hembras infectadas. Por cada cepa y concentración de la solución fungosa evaluada, se tomaron aleatoriamente cinco hembras muertas con presencia de conidios del hongo, se colocaron en forma individual en viales de vidrio con $2 \mathrm{~mL}$ de agua destilada $+0.01 \%$ de glicerina, posteriormente cada tubo fue homogenizado en un vortex durante dos minutos para desprender los conidios del cuerpo del insecto. De esta solución se realizaron las diluciones de $10^{-1}$ a $10^{-4} \mathrm{y}$ utilizando un hemocitómetro se cuantificó el número de conidios por mosca de cada concentración y cepa con que fueron tratadas. Por cada cepa se realizó un diseño experimental con una distribución completamente al azar, en donde las concentraciones del hongo fueron los tratamientos y las moscas infectadas se consideraron las repeticiones, dando un total de 50 unidades experimentales por cepa.

Análisis estadístico. Para determinar el $\mathrm{TL}_{50}$ y $\mathrm{CL}_{50}$ de cada cepa evaluada, los datos de mortalidad obtenidos en cada concentración mediante los bioensayos fueron corregidos a partir de la mortalidad natural registrada en los insectos del testigo, utilizando la fórmula de Abbott (1925). Posteriormente fueron analizados utilizando un modelo Probit (Pc Probit, SAS Institute 1992). Los valores de $\mathrm{CL}_{50}$ 's obtenidos de las cinco cepas con mayor agresividad fueron sometidas a un análisis de varianza (ANOVA). La separación de medias se realizó mediante la prueba de Tukey $(P>$ 0.05) (SAS Institute 1992).

Los datos de porcentajes de moscas infectadas por el hongo y con presencia de conidios fueron transformados con la formula Arc. Sen $\sqrt{ } X$ para cumplir con los supuestos de normalidad y homogenidad de la varianza (Little \& Jackson 1989). Posteriormente fueron sometidos a un análisis de varianza (ANOVA) y la separación de medias se hizo utilizando la prueba de Tukey $(P>0.05)$ (SAS Institute 1992).

Los datos de la cantidad de conidios recuperados de las hembras muertas e infectadas, tratadas con las concentraciones de cada cepa fueron sometidos a un análisis de varianza (ANOVA). La separación de medias se realizó mediante la prueba de Tukey $(P>0.05)$ (SAS Institute 1992). 


\section{RESULTADOS}

Patogenicidad a hembras. En la prueba de patogenicidad en donde fueron evaluadas las 16 cepas del hongo Beauveria bassiana contra hembras de Ceratitis capitata, se comprobó que todas fueron patogénicas a dicha fase biológica del insecto, registrándose un rango de mortalidad corregida (moscas infectadas) de 9.1 a 91.9\% (Cuadro 2). Las diferencias observadas entre los valores promedios de mortalidad ocasionada por cada cepa fueron significativas (prueba de Tukey, $P>0.05$ ).

Las cepas con mayor virulencia fueron la Bb25, Bb6, Bb26, Bb17 y la Bb15, ya que ocasionaron la mayor mortalidad en las hembras que fueron tratadas. Las cepas $\mathrm{Bb} 27, \mathrm{Bb} 4$ y Bb18 tuvieron una virulencia intermedia, con mortalidades de $58.0 \mathrm{a}$ $65.2 \%$. Las cepas que registraron una menor virulencia fueron aquellas que ocasionaron promedios menores al 50\% de mortalidad. La mortalidad en las moscas del testigo fue de $12.9 \pm 2.8 \%$, considerada dentro de los límites aceptables en estudios con entomopatógenos.

Cuadro 2. Mortalidad de hembras de Ceratitis capitata por diversas cepas del hongo entomopatógeno Beauveria bassiana en condiciones de laboratorio.

\begin{tabular}{lcc}
\hline Cepa & Concentración $($ Conidios $/ \mathbf{m L})$ & Mortalidad $(\% \pm \mathbf{E E}) *$ \\
\hline Bb25 & $1.7 \times 10^{8}$ & $91.9 \pm 1.1 \mathrm{a}$ \\
Bb6 & $4.5 \times 10^{8}$ & $91.2 \pm 1.2 \mathrm{a}$ \\
Bb26 & $2.0 \times 10^{8}$ & $87.4 \pm 2.4 \mathrm{ab}$ \\
Bb17 & $6.5 \times 10^{8}$ & $71.0 \pm 9.1 \mathrm{bc}$ \\
Bb15 & $1.1 \times 10^{8}$ & $70.2 \pm 11.8 \mathrm{bc}$ \\
Bb18 & $2.5 \times 10^{8}$ & $65.2 \pm 9.8 \mathrm{~cd}$ \\
Bb4 & $2.5 \times 10^{8}$ & $60.5 \pm 3.7 \mathrm{cde}$ \\
Bb27 & $1.0 \times 10^{8}$ & $58.0 \pm 2.9 \mathrm{def}$ \\
Bb13 & $1.0 \times 10^{8}$ & $48.9 \pm 3.8 \mathrm{efg}$ \\
Bb12 & $2.8 \times 10^{8}$ & $38.8 \pm 19.2 \mathrm{fg}$ \\
Bb8 & $1.3 \times 10^{8}$ & $37.9 \pm 0.8 \mathrm{fg}$ \\
Bb7 & $3.3 \times 10^{8}$ & $36.6 \pm 4.8 \mathrm{gh}$ \\
Bb16 & $2.6 \times 10^{8}$ & $18.5 \pm 7.5 \mathrm{gh}$ \\
Bb9 & $1.6 \times 10^{8}$ & $15.3 \pm 11.0 \mathrm{~h}$ \\
Bb19 & $2.4 \times 10^{8}$ & $14.2 \pm 5.5 \mathrm{~h}$ \\
Bb2 & $2.1 \times 10^{8}$ & $9.1 \pm 1.5 \mathrm{~h}$ \\
Testigo & - & $12.9 \pm 2.8+$ \\
\hline
\end{tabular}

\footnotetext{
* Mortalidad corregida por la fórmula de Abbot. EE = Error estándar. +No incluido para el análisis de varianza realizado con moscas infectadas. Los valores promedios de mortalidad seguidos por una misma letra no son significativamente diferentes (prueba de Tukey, $P>0.05$ ).
} 
Determinación del tiempo letal medio $\left(\mathbf{T L}_{\mathbf{5 0}}\right)$. Las cepas $\mathrm{Bb6}$ y la $\mathrm{Bb} 19$ registraron el menor y mayor tiempo letal medio, respectivamente (Cuadro 3). De acuerdo a los valores de los límites fiduciales al $95 \%$ de las cepas evaluadas, las diferencias de los $\mathrm{TL}_{50}$ 's fueron significativas $(P<0.001)$, clasificados en cinco categorías; el primer grupo (a) correspondió a 3.83 días para la cepa Bb6; el segundo grupo (ab) fue de 3.91 a 4.52 días (cepas Bb15, Bb17, Bb25, Bb26 y Bb18), el tercer grupo (bc) fue de 5.15 a 5.81 días (cepas Bb12 y Bb8), en el cuarto grupo (c) con rangos de 6.19 a 11.47 días se incluyen las cepas $\mathrm{Bb} 4, \mathrm{Bb} 13, \mathrm{Bb} 7, \mathrm{Bb} 9, \mathrm{Bb} 27, \mathrm{Bb} 16$, $\mathrm{Bb} 2$, y el quinto grupo (d) con 17.64 días correspondió a la cepa Bb19.

Cuadro 3. Tiempos letales medios $\left(\mathrm{TL}_{50}\right)$ determinados en hembras de Ceratitis capitata tratadas con soluciones fungosas de diferentes cepas de Beauveria bassiana en condiciones de laboratorio.

\begin{tabular}{lc}
\hline Cepa & TL $_{50}$ (Días, Límites fiduciales al 95\%)* \\
\hline Bb 6 & $3.83(3.53-4.11) \mathrm{a}$ \\
$\mathrm{Bb} 15$ & $3.91(3.49-4.57) \mathrm{ab}$ \\
$\mathrm{Bb} 17$ & $3.97(3.58-4.54) \mathrm{ab}$ \\
$\mathrm{Bb} 25$ & $4.01(3.70-4.31) \mathrm{ab}$ \\
$\mathrm{Bb} 26$ & $4.22(3.98-4.46) \mathrm{ab}$ \\
$\mathrm{Bb} 18$ & $4.52(4.08-5.30) \mathrm{ab}$ \\
$\mathrm{Bb} 12$ & $5.15(4.12-9.39) \mathrm{bc}$ \\
$\mathrm{Bb} 8$ & $5.81(4.55-9.52) \mathrm{bc}$ \\
$\mathrm{Bb} 4$ & $6.19(5.70-9.78) \mathrm{c}$ \\
$\mathrm{Bb} 13$ & $6.20(5.43-12.83) \mathrm{c}$ \\
$\mathrm{Bb} 7$ & $6.25(5.77-12.02) \mathrm{c}$ \\
$\mathrm{Bb} 9$ & $8.40(6.12-16.74) \mathrm{c}$ \\
$\mathrm{Bb} 27$ & $8.63(6.10-21.46) \mathrm{c}$ \\
$\mathrm{Bb} 16$ & $10.33(7.61-19.24) \mathrm{c}$ \\
$\mathrm{Bb} 2$ & $11.47(8.19-23.08) \mathrm{c}$ \\
$\mathrm{Bb} 19$ & $17.64(10.56-41.19) \mathrm{d}$ \\
\hline
\end{tabular}

\footnotetext{
* Valores seguidos con la misma letra no hay diferencia significativa de acuerdo a sus límites fiduciales al 95\%.
}

Concentración letal media $\left(\mathbf{C L}_{\mathbf{5 0}}\right)$. En esta fase la cepa $\mathrm{Bb} 25$ fue la que registró mayor agresividad al ocasionar la mayor mortalidad en menor tiempo; le siguió la cepa Bb6 y la cepa Bb17 tuvo una agresividad intermedia. Las cepas con menor agresividad fueron $\mathrm{Bb} 26$ y la $\mathrm{Bb} 15$. En base a estos resultados y considerando el traslape de sus límites fiduciales, se pueden clasificar en dos 
grupos; el primer grupo corresponde a las cepas $\mathrm{Bb} 25, \mathrm{Bb} 6, \mathrm{Bb} 17$ y $\mathrm{Bb} 26$, y un segundo grupo conformado por las cepas $\mathrm{Bb} 26$ y Bb15. La cepa Bb26 se ubica en ambos grupos debido a que sus límites fiduciales al 95\% se traslapan con el primero y segundo grupo (Cuadro 4).

Cuadro 4. Patogenicidad de cinco cepas del hongo entomopatógeno Beauveria bassiana en hembras de Ceratitis capitata en condiciones de laboratorio.

\begin{tabular}{llllll}
\hline Cepa & $\begin{array}{l}\text { Concentración } \\
\text { (Conidios/mL) }\end{array}$ & $\begin{array}{l}\text { CL50 (Límites }, \\
\text { fiduciales, 95\%)* }\end{array}$ & CL $_{\mathbf{9 5}}$ & $\mathbf{X}^{\mathbf{2}}$ & $\begin{array}{c}\text { Ecuación de la } \\
\text { línea de regresión }\end{array}$ \\
\hline $\mathrm{Bb} \mathrm{25}$ & $1.06 \times 10^{5}$ & $0.017(0.011-0.025) \mathrm{a}$ & 9.213 & 3.0 & $\mathrm{Y}=6.29+0.31 \mathrm{x}$ \\
$\mathrm{Bb} \mathrm{6}$ & $3.94 \times 10^{5}$ & $0.021(0.014-0.029) \mathrm{a}$ & 5.063 & 4.4 & $\mathrm{Y}=6.39+0.35 \mathrm{x}$ \\
$\mathrm{Bb} \mathrm{17}$ & $5.75 \times 10^{5}$ & $0.030(0.021-0.048) \mathrm{a}$ & 8.522 & 4.0 & $\mathrm{Y}=6.29+0.36 \mathrm{x}$ \\
$\mathrm{Bb} \mathrm{26}$ & $1.80 \times 10^{6}$ & $0.042(0.023-0.074) \mathrm{ab}$ & 66.09 & 6.0 & $\mathrm{Y}=5.74+0.23 \mathrm{x}$ \\
$\mathrm{Bb} \mathrm{15}$ & $8.72 \times 10^{6}$ & $0.054(0.031-0.096) \mathrm{b}$ & 196.28 & 2.6 & $\mathrm{Y}=5.62+0.21 \mathrm{x}$
\end{tabular}

* Los valores seguidos con la misma letra no hay diferencia significativa de acuerdo a sus limites fiduciales al $95 \%$.

Esporulación de B. bassiana en hembras infectadas. Como era de esperarse se observó una reducción gradual en la producción de conidios a partir de las moscas muertas que fueron tratadas con las menores concentraciones que las tratadas con las concentraciones mayores. La mayor producción de conidios se observó en las moscas que fueron tratadas con la concentración de 1.0\% de las cepas Bb6 y Bb17; seguido de las cepas $\mathrm{Bb} 15$ y la cepa $\mathrm{Bb} 25$, y la cepa que produjo una menor cantidad de conidios fue la $\mathrm{Bb} 26$. El menor porcentaje de producción de conidios se registró en las moscas tratadas con la concentración de $0.001 \%$; con esta concentración la cepa $\mathrm{Bb} 15$ fue la que produjo mayor cantidad de conidios, le siguieron las cepas $\mathrm{Bb} 26$ y Bb25; las cepas que produjeron menor cantidad de conidios fueron la $\mathrm{Bb} 6 \mathrm{y}$ la Bb17 (Cuadro 5). Las diferencias observadas entre los porcentajes de conidios producidos entre las concentraciones de una misma cepa sí fueron significativas: cepa Bb6 $(F=67.0 ;$ g.1. $=9,20 ; P<0.001)$, cepa Bb15 $(F=29.5 ;$ g.1. $=9,20 ; P<$ $0.001)$, cepa Bb17 $(F=77.8 ;$ g.l. $=9,20 ; P<0.001)$, cepa Bb25 $(F=59.0 ;$ g.l. $=9$, 20; $P<0.001)$, cepa Bb26 $(F=57.9 ;$ g.l. $=9,20 ; P<0.001)$. En este ocasión, las diferencias observadas sí fueron significativas $(P>0.05)$.

Sin embargo, al comparar la producción de conidios entre cepas, solo los porcentajes de conidios producidos en las dos concentraciones menores de la cepa Bb15 fueron significativamente mayores a la de las otras cepas: concentración 
$0.003 \%(F=4.9$; g.l. $=4,10 ; P=0.019)$, concentración $0.001 \%(F=5.6$; g.l. $=4$, $10 ; P=0.012)$. En las otras concentraciones, las diferencias observadas entre los valores a las mismas concentraciones no fueron significativas $(P<0.05)$.

Recuperación de conidios a partir de moscas infectadas. A medida que las moscas fueron tratadas con menores concentraciones, también la cantidad de conidios recuperados disminuyó de manera gradual para cada cepa. La mayor cantidad de conidios se recuperó a partir de las moscas tratadas con la cepa Bb6 a la concentración de 1.0\%, seguidos por las cepas Bb26 y Bb15; y de las moscas tratadas con las cepas Bb25 y Bb17 se recuperó una menor cantidad de conidios. Con la concentración de $0.001 \%$, se recuperó menor cantidad de conidios en las moscas tratadas con las cinco cepas. Aunque se observó la misma tendencia, es decir que en las moscas tratadas con la cepa Bb6 se recuperó mayor cantidad de conidios, le siguieron aquellas que fueron tratadas con las cepas $\mathrm{Bb} 26, \mathrm{Bb} 15, \mathrm{Bb} 25$ y la menor cantidad fue a partir de las moscas tratadas con la cepa Bb17 (Cuadro 6). Las diferencias observadas entre las cantidades de conidios recuperados a partir de las moscas tratadas con las diferentes concentraciones sí fueron significativas: cepa Bb6 $(F=10.11 ;$ g.l. $=9,40 ; P<0.001)$, cepa Bb15 $(F=5.30 ;$ g.l. $=9,40 ; P<0.001)$, cepa Bb17 $(F=19.25$; g.l. $=9,40 ; P<0.001)$, cepa Bb25 $(F=13.71 ;$ g.l. $=9,40 ; P$ $<0.001)$ у сера Bb26 $(F=5.96 ;$ g.l. $=9,40 ; P<0.001)$ (Cuadro 6).

Cuadro 5. Esporulación ( $\% \pm \mathrm{EE})$ de hembras de Ceratitis capitata infectadas, previamente tratadas con diferentes concentraciones del hongo Beauveria bassiana.

\begin{tabular}{cccccc}
\hline Concentración & \multicolumn{5}{c}{ Cepas del hongo* } \\
\cline { 2 - 6 }$(\%)$ & Bb6 & Bb15 & Bb17 & Bb25 & Bb26 \\
\hline & $68.0 \pm 2.3 \mathrm{~A}$ & $66.4 \pm 2.0 \mathrm{~A}$ & $68.0 \pm 2.9 \mathrm{~A}$ & $66.4 \pm 2.0 \mathrm{~A}$ & $64.9 \pm 2.8 \mathrm{~A}$ \\
1.0 & $64.9 \pm 1.7 \mathrm{~A}$ & $60.7 \pm 2.5 \mathrm{AB}$ & $62.0 \pm 1.7 \mathrm{~B}$ & $62.0 \pm 2.3 \mathrm{AB}$ & $60.7 \pm 1.4 \mathrm{AB}$ \\
0.6 & $63.4 \pm 1.5 \mathrm{~A}$ & $55.6 \pm 2.2 \mathrm{~B}$ & $56.8 \pm 0.7 \mathrm{~B}$ & $58.1 \pm 2.2 \mathrm{~B}$ & $58.1 \pm 2.2 \mathrm{~B}$ \\
0.3 & $50.8 \pm 3.3 \mathrm{~B}$ & $47.3 \pm 2.5 \mathrm{C}$ & $50.8 \pm 3.3 \mathrm{C}$ & $49.6 \pm 2.6 \mathrm{C}$ & $45.0 \pm 2.1 \mathrm{C}$ \\
0.1 & $45.0 \pm 2.3 \mathrm{BC}$ & $43.9 \pm 2.2 \mathrm{C}$ & $43.9 \pm 1.7 \mathrm{D}$ & $46.2 \pm 1.6 \mathrm{C}$ & $34.5 \pm 2.8 \mathrm{D}$ \\
0.06 & $40.4 \pm 1.4 \mathrm{CD}$ & $42.7 \pm 1.3 \mathrm{CD}$ & $38.1 \pm 1.7 \mathrm{D}$ & $38.1 \pm 1.2 \mathrm{D}$ & $32.0 \pm 1.7 \mathrm{DE}$ \\
0.03 & $36.9 \pm 1.2 \mathrm{DE}$ & $36.9 \pm 2.2 \mathrm{DE}$ & $30.7 \pm 2.0 \mathrm{E}$ & $36.9 \pm 2.2 \mathrm{D}$ & $29.3 \pm 2.2 \mathrm{DEF}$ \\
0.01 & $30.7 \pm 3.3 \mathrm{E}$ & $34.5 \pm 2.4 \mathrm{EF}$ & $25.1 \pm 1.6 \mathrm{EF}$ & $32.0 \pm 2.1 \mathrm{D}$ & $26.6 \pm 2.6 \mathrm{EFG}$ \\
0.006 & $22.0 \pm 1.7 \mathrm{~F} \mathrm{~b}$ & $32.0 \pm 2.9 \mathrm{EF}$ a & $22.0 \pm 1.7 \mathrm{FG} \mathrm{b}$ & $25.1 \pm 1.2 \mathrm{E} \mathrm{b}$ & $25.1 \pm 1.2 \mathrm{FG} \mathrm{b}$ \\
0.003 & $16.4 \pm 2.1 \mathrm{~F} \mathrm{~b}$ & $28.0 \pm 2.3 \mathrm{~F} \mathrm{a}$ & $16.4 \pm 1.5 \mathrm{G} \mathrm{b}$ & $18.4 \pm 2.3 \mathrm{~F} \mathrm{~b}$ & $20.3 \pm 1.8 \mathrm{G} \mathrm{b}$ \\
0.001 & & & & \\
\hline
\end{tabular}

\footnotetext{
* Los valores promedios en cada columna seguidos por una misma letra (mayúscula) no son significativamente diferentes (prueba de Tukey, $P>0.05$ ). Los valores promedios en cada fila seguidos por una misma letra (minúscula) no son significativamente diferentes (prueba de Tukey, $P>0.05$ ). EE = error estándar.
} 
Muñoz et al.: Mortalidad de Ceratitis capitata por Beauveria bassianaActa Zoológica Mexicana (n.s.) 25(3) (2009)

Cuadro 6. Cantidad de conidios producidos por cinco cepas de Beauveria bassiana en hembras infectadas de Ceratitis capitata, previamente tratadas con diferentes concentraciones.

$(N=$ Promedio $( \pm$ EE) de cinco moscas /concentración / cepa).

\begin{tabular}{cccccc}
\hline $\begin{array}{c}\text { Concentración } \\
(\%)\end{array}$ & Bb6 & Bb15 & Bb17 & Bb25 & Bb26 \\
\cline { 2 - 6 } & & & & & \\
\hline \multirow{2}{*}{1.0} & $3.65 \pm 1.01 \times 10^{8} \mathrm{a}$ & $1.23 \pm 0.60 \times 10^{8} \mathrm{a}$ & $5.58 \pm 1.12 \times 10^{7} \mathrm{a}$ & $6.14 \pm 1.42 \times 10^{7} \mathrm{a}$ & $3.11 \pm 0.37 \times 10^{8} \mathrm{a}$ \\
0.6 & $2.19 \pm 0.59 \times 10^{8} \mathrm{ab}$ & $7.41 \pm 3.55 \times 10^{7} \mathrm{ab}$ & $3.35 \pm 0.67 \times 10^{7} \mathrm{ab}$ & $3.68 \pm 0.87 \times 10^{7} \mathrm{~b}$ & $1.86 \pm 0.22 \times 10^{8} \mathrm{~b}$ \\
0.3 & $1.09 \pm 0.30 \times 10^{8} \mathrm{bc}$ & $3.70 \pm 1.80 \times 10^{7} \mathrm{~b}$ & $1.67 \pm 0.33 \times 10^{7} \mathrm{abc}$ & $1.84 \pm 0.72 \times 10^{7} \mathrm{bc}$ & $9.33 \pm 1.47 \times 10^{7} \mathrm{c}$ \\
0.1 & $3.65 \pm 1.00 \times 10^{7} \mathrm{bc}$ & $1.23 \pm 0.58 \times 10^{7} \mathrm{~b}$ & $5.58 \pm 0.11 \times 10^{6} \mathrm{bc}$ & $6.14 \pm 1.40 \times 10^{6} \mathrm{c}$ & $3.11 \pm 0.37 \times 10^{7} \mathrm{~cd}$ \\
0.06 & $2.19 \pm 0.58 \times 10^{7} \mathrm{c}$ & $7.41 \pm 3.50 \times 10^{6} \mathrm{~b}$ & $3.35 \pm 0.67 \times 10^{6} \mathrm{bc}$ & $3.68 \pm 0.90 \times 10^{6} \mathrm{c}$ & $1.86 \pm 0.23 \times 10^{7} \mathrm{~cd}$ \\
0.03 & $1.09 \pm 0.30 \times 10^{7} \mathrm{c}$ & $3.70 \pm 1.80 \times 10^{6} \mathrm{~b}$ & $1.67 \pm 0.33 \times 10^{6} \mathrm{c}$ & $1.84 \pm 0.72 \times 10^{6} \mathrm{c}$ & $9.33 \pm 0.16 \times 10^{6} \mathrm{~cd}$ \\
0.01 & $3.65 \pm 1.00 \times 10^{6} \mathrm{c}$ & $1.23 \pm 0.59 \times 10^{6} \mathrm{~b}$ & $5.58 \pm 1.12 \times 10^{5} \mathrm{c}$ & $6.14 \pm 1.37 \times 10^{5} \mathrm{c}$ & $3.11 \pm 0.34 \times 10^{6} \mathrm{~d}$ \\
0.006 & $2.19 \pm 0.58 \times 10^{6} \mathrm{c}$ & $7.41 \pm 3.50 \times 10^{5} \mathrm{~b}$ & $3.35 \pm 0.67 \times 10^{5} \mathrm{c}$ & $3.68 \pm 0.85 \times 10^{5} \mathrm{c}$ & $1.86 \pm 0.13 \times 10^{6} \mathrm{~d}$ \\
0.003 & $1.09 \pm 0.30 \times 10^{6} \mathrm{c}$ & $3.70 \pm 1.80 \times 10^{5} \mathrm{~b}$ & $1.67 \pm 0.33 \times 10^{5} \mathrm{c}$ & $1.84 \pm 0.69 \times 10^{5} \mathrm{c}$ & $9.33 \pm 1.10 \times 10^{5} \mathrm{~d}$ \\
0.001 & $3.65 \pm 1.00 \times 10^{5} \mathrm{c}$ & $1.23 \pm 0.64 \times 10^{5} \mathrm{~b}$ & $5.58 \pm 1.12 \times 10^{4} \mathrm{c}$ & $6.14 \pm 1.38 \times 10^{4} \mathrm{c}$ & $3.11 \pm 0.50 \times 10^{5} \mathrm{~d}$ \\
\hline
\end{tabular}

* Los valores promedios de cada columna seguidos por una misma letra no son significativamente diferentes (prueba de Tukey, $P>0.05$ ). EE = error estándar.

\section{DISCUSIÓN}

Mediante bioensayos realizados en laboratorio se demostró que varias cepas de $B$. bassiana que están depositadas en el cepario de ECOSUR son patogénicas a hembras de $C$. capitata. Los resultados obtenidos coinciden con otros previamente realizados con moscas de la fruta, ya sea utilizando estas mismas cepas cuando fueron evaluadas contra adultos de Anastrepha ludens (Loew), en donde se registraron mortalidades de 82 a 100\% (De la Rosa et al. 2002). Otras cepas de $B$. bassiana han sido evaluadas contra adultos de tres especies del género Ceratitis $[C$. capitata, C. cosyra (Walker) y C. rosa (Karsch)] con mortalidades similares a los reportados en nuestro estudio (Dimbi et al. 2003). En cambio con algunas cepas de Metarhizium anisopliae evaluados contra adultos de C. capitata se obtuvieron mortalidades entre 69 y $78 \%$ (Espin et al. 1989), que son menores a los ocasionados por B. bassiana. Pero también se han caracterizado cepas de $M$. anisopliae que tienen alta virulencia contra adultos de $C$. capitata y otras especies de este mismo género (Ekesi et al. 2002, Dimbi et al. 2003).

En adultos de otros dípteros ciclórrafos como la mosca tse-tse (Glossinia morsitans morsitans), se han reportado mortalidades hasta de 97\% (Kaaya \& Munyinyi 1995). En adultos de mosca casera, Musca domestica L. (Diptera: Muscidae), las mortalidades ocasionadas por B. bassiana han variado de 94 a 100\% (Barson et al. 
1994). También cuando se evaluaron bajo condiciones de laboratorio, seis especies de hongos entomopatógenos (Beauveria bassiana, B. brongniartii, Metarhizium anisopliae, Tolypocladium cylindrosporum, Paecilomyces farinosus y Verticillium lecanii) contra la mosca casera (Musca domestica L.) demostrando en este caso que las hembras fueron más susceptibles al ataque de B. bassiana, ya que se registraron mortalidades del 100\%; caso contrario a lo observado con las otras especies de hongos en donde el efecto sobre el insecto fue menor (Barson et al. 1994).

De acuerdo a los resultados obtenidos en este trabajo y a lo reportado por De la Rosa et al. (2002), cuando evaluaron las mismas cepas contra A. ludens, la mortalidad fue similar a la registrada por la mayoría de las cepas en este estudio, solamente en algunas cepas hubieron diferencias. Existen numerosos reportes indicando que las diferencias en la susceptibilidad de los insectos hospederos hacia un determinado entomopatógeno. Estas diferencias entre colonias, razas, cepas o poblaciones de insectos son debidas a las prolongadas asociaciones del hospedero con el patógeno que provocan un proceso de selección natural (Lecuona et al. 1996). Los factores endógenos y exógenos están relacionados con la tolerancia o susceptibilidad pero los efectos no son claros, ya que actúan en una asociación entre el insecto y el patógeno, de tal forma que los factores endógenos (fisiológicos y genéticos) se asocian e influyen en la susceptibilidad del insecto hospedero; sin embargo, estos pueden ser modificados por factores exógenos como la temperatura y la alimentación del insecto hospedero (Watanabe 1987).

La agresividad de una cepa está determinada en base al tiempo letal medio $\left(\mathrm{LT}_{50}\right)$, considerándose una cepa con mayor agresividad y virulenta aquella que mata a su hospedero en menor tiempo (De la Rosa et al. 2002). Las diferencias en los tiempos para matar al $50 \%$ de la población del hospedero han sido variables y están en función de la virulencia de la cepa del hongo entomopatógeno, del hospedero de donde fue aislado el patógeno, de la edad y del sexo del hospedero en que se está evaluando (Hajek \& St. Leger 1994, Maniania \& Ondulaja 1998). Por lo tanto, en los estudios se ha observado variabilidad en los resultados, como es el caso del hongo $B$. bassiana contra adultos de A. ludens que se obtuvieron tiempos letales de 2.82 a 5.99 días (De la Rosa et al. 2002) y con cepas de M. anisopliae aplicados a adultos de $C$. capitata, se reportó un $\mathrm{TL}_{50}$ de 11 días (Espín et al. 1989). Pero los resultados obtenidos con otras especies de tefrítidos, la respuesta ha sido similar a lo observado en nuestra investigación, y con otras especies de dípteros como Musca domestica (L.) y la mosca tse-tse, Glosinia morsitans, registrando mortalidades hasta del 100\%, 10 días después que los insectos fueron tratados (Kaaya et al. 1991, Watson et al. 1995).

Solamente los valores de tiempos letales registrados por algunas cepas de $B$. bassiana fueron diferentes entre A. ludens y $C$. capitata, estas diferencias puede ser atribuible a una serie de interacciones entre la edad, la especie y el estado fisiológico de ambas especies de moscas de la fruta (Hajek \& St. Leger 1994, De la Rosa et al. 
2002, Ekesi et al. 2002). Dichos factores también pudieron haber sido influenciados por factores físicos como la temperatura y la humedad relativa, que en ocasiones modifican la expresión de la virulencia del patógeno como se ha indicado en estudios con otros insectos hospederos (Hajek \& St. Leger 1994, García-Gutiérrez et al. 2007).

Otra de las causas que marcan la diferencia en la virulencia de las cepas del hongo son las enzimas degradadoras de la cutícula que producen durante la penetración al interior del hospedero (Samuels \& Paterson 1995). Beauveria bassiana durante este proceso produce las siguientes enzimas: proteinazas, lipasas, glicogenasas y quimoestelasas, que intervienen en la degradación de la cutícula del hospedero, actividad que tiene una relación estrecha con una muerte rápida de los insectos infectados (Hall 1982, Lecuona et al. 1996, Samuels \& Paterson 1995).

En el caso de $C$. capitata, una vez que los insectos murieron, la mayoría de los órganos y tejidos fueron invadidos por el crecimiento micelial, después ocurrió una invasión total por parte del hongo y el cadáver adquirió un aspecto de momificación, tolerante a la descomposición bacteriana. Esta sintomatología ya ha sido descrita en otros hospederos muertos e infectados por diversas cepas de dicho hongo (Hajek \& St. Leger 1994, De la Rosa et al. 2002). La presencia del hongo en el interior del hospedero adquiere el aspecto de una masa de micelios que mantiene intacto al integumento, pero cuando las condiciones de humedad son adecuadas, los micelios atraviesan el integumento del interior hacia el exterior del insecto, emergiendo las hifas generalmente en las partes suaves y menos esclerotizadas (Hajek \& St. Leger 1994, García-Gutiérrez et al. 2007). A pesar de que algunos insectos son altamente susceptibles a la acción infectiva de los hongos entomopatógenos, el desarrollo del hongo sobre el cuerpo de los insectos infectados es posible solamente cuando las condiciones de humedad relativa son óptimas (Hajek \& St. Leger 1994, Lecuona et al. 1996). Después que las hifas atravesaron el integumento, el hongo inició la etapa vegetativa y de ahí pasó a la etapa reproductiva formando los conidios, si las condiciones de humedad son óptimas, la cantidad de conidios producidos varia de acuerdo con la especie, tamaño del hospedero y obviamente con la cepa del patógeno (De la Rosa et al. 2002). El proceso de esporulación del patógeno sobre los insectos infectados también varía de acuerdo con el hospedero de donde se obtuvo la cepa y es un factor importante en la diseminación y ocurrencia de la enfermedad en la población de insectos hospederos bajo condiciones de campo (García-Gutiérrez et al. 2007). El insecto infectado adquiere una coloración característica de acuerdo a la especie de hongo, una vez que se ha multiplicado y esporulado, los conidios son diseminados y están distribuidos de manera constante en el ambiente para infectar a nuevos hospederos que en ocasiones puede producir a nivel de campo, epizootias con potencial para contribuir a la regulación de una determinada población de insectos plaga (Lecuona et al. 1996, Tanada 1963). 
Los resultados obtenidos en nuestro trabajo concuerdan con los reportados por otros investigadores y considerando los bajos valores correspondientes a los tiempos letales medios $\left(\mathrm{TL}_{50}\right)$ determinados para las cepas que expresaron mayor virulencia, pero como $C$. capitata es una plaga de interés cuarentenario para México, en esta investigación se utilizaron moscas irradiadas, por lo que podrían ser más susceptibles a la infección del hongo. Por lo tanto, se sugiere que las cepas que tuvieron mayor virulencia sean evaluadas contra moscas fértiles o silvestres, para confirmar su potencial antes de ser evaluadas en condiciones de campo. De confirmarse su virulencia contra moscas fértiles, el uso de alguna de las cepas con mayor actividad biológica representa una alternativa más como parte complementaria en el control de dicha plaga y otras especies de moscas de la fruta, que provocan severos daños a la fruticultura. Por lo tanto, se sugiere realizar futuras investigaciones para desarrollar los mecanismos que permitan su aplicación en los programas de manejo que se llevan a cabo contra dicha plaga en huertos de frutales y plantaciones de café, sobretodo en aquellos sistemas de producción que tienen un enfoque orgánico.

Agradecimientos: Este trabajo está dedicado a la memoria del M.C. William de la Rosa (†), quién formó parte del equipo pionero del proyecto: "Control microbiano de moscas de la fruta" en ECOSUR. Se agradece el apoyo del Laboratorio de Cría del Programa Moscamed (SAGARPA) por el material biológico proporcionado.

\section{LITERATURA CITADA}

Abbott, W. S. 1925. A method of computing the effectiveness of an insecticide. Journal of Economic Entomology, 18: 265-267.

Barson, G., N. Renn \& F. A. Bywater. 1994. Laboratory evaluation of six species of entomopathogenic fungi for the control of the house fly (Musca domestica L.) a pest of intensive animal units. Journal of Invertebrate Pathology, 64: 107-113.

Castillo, M. A., P. Moya, E. Hernández \& E. Primo-Yúfera. 2000. Susceptibility of Ceratitis capitata Wiedemann (Diptera: Tephritidae) to entomopathogenic fungi and their extracts. Biological Control, 19: 274-282.

De la Rosa, W., F. L. López \& P. Liedo. 2002. Beauveria bassiana as a pathogen of the Mexican fruit fly (Diptera: Tephritidae) under laboratory conditions. Journal of Economic Entomology, 95: 36-43.

Dimbi, S., N. K. Maniania, S. A. Lux, S. Ekesi \& J. K. Mueke. 2003. Pathogenicity of Metarhizium anisopliae (Metsch.) Sorokin and Beauveria bassiana (Balsamo) Vuillemin, to three adult fruit fly species: Ceratitis capitata (Wiedemann), C. rosa var. fasciventris Karsch and C. cosyra (Walker) (Diptera: Tephritidae). Mycopathologia, 156: 375-382.

Domínguez-Gordillo, J. C., D. Castellanos-Hernández, E. Hernández-Ortiz \& A. MartínezGrajales. 2000. Métodos de cría masiva de moscas de la fruta. Pp. 399-414. In: Memorias del XIII Curso Internacional sobre Moscas de la Fruta. Centro Internacional de Capacitación en Moscas de la Fruta. Metapa de Domínguez, Chiapas, México.

Ekesi, S., N. K. Maniania \& S. A. Lux. 2002. Mortality in three African tephritid fruit fly puparia and adults caused by the entomopathogenic fungi Metarhizium anisopliae and Beauveria bassiana. Biocontrol Science and Technology, 12: 7-17. 
Ekesi, S., N. K. Maniania, S. A. Mohamed \& S. A. Lux. 2005. Effect of soil application of Metarhizium anisopliae on African tephritid fruit flies and their associated endoparasitoids. Biological Control, 35: 83-91.

Enkerlin, D., R. L. Garcia \& F. M. Lopez. 1989. Mexico, Central and South America. Pp. 83-90. In: A. S. Robinson and G. Hooper (Eds). Fruit flies: Their biology, natural enemies and control. Vol. 3A. World Crop Pests. Elsevier. Amsterdam, The Netherlands.

Espin, G. A. T., H. M. L. Souza, C. L. Messias \& A. E. Piedrabuena. 1989. Pateginicidade de Metarhizium anisopliae nas diferentes fases de desenvolvimiento de Ceratitis capitata (Wied.) (Diptera: Tephritidae). Revista Brasileira de Entomologia, 33: 17-23.

García, A. S., C. L. Messias, H. M. L. Souza \& A. E. Piedrabuena. 1984. Patoginicidade de Metarhizium anisopliae var. anisopliae a Ceratitis capitata (Wiedemann) (Diptera: Tephritidae). Revista Brasileira de Entomologia, 28: 421-424.

García-Gutiérrez, C., V. M. Hernández-Velásquez \& M. B. González-Maldonado. 2007. Hongos entomopatógenos. Pp. 91-118. In: C. García-Gutiérrez y H. Medrano-Roldán (Eds). Biotecnología financiera aplicada a bioplaguicidas. Edit. Artes Graficas La Impresora. Durango, México.

Gutiérrez, S. J. 1976. La mosca del mediterráneo Ceratitis capitata (Wied.) y los factores ecológicos que favorecerían su establecimiento y propagación en México. Dirección General de Sanidad Vegetal. Secretaría de Agricultura y Ganadería. México, D. F.

Hajek, A. E. \& R. J. St. Leger. 1994. Interactions between fungal pathogens and insect hosts. Annual Review of Entomology, 39: 293-322.

Hall, R. A. 1982. Deuteromycetes virulence and bioassay design. Pp. 191-196. In: Invertebrate pathology and microbial control. Proceeding of the 3rd. International Colloquium on Invertebrate Pathology. University of Sussex. London, UK.

Hendrichs, J., A. S. Robinson, J. P. Cayol \& W. Enkerlin. 2002. Medfly areawide sterile insect technique programmes for prevention, supression or erradication: The importance of mating behavior studies. Florida Entomologist, 85: 1-13.

Jiménez, J. A. 1992. Patogenicidad de diferentes aislamientos de Beauveria bassiana sobre la broca del café. Cenicafe, 43: 84-98.

Kaaya, G. P., E. D. Kokwaro \& J. K. Murithi. 1991. Mortalities in adult Glossina morsitans morsitans experimentally infected with the entomogenous fungi Beauveria bassiana and Metarhizium anisopliae. Discovery and Innovation, 3: 55-60.

Kaaya, G. P. \& M. D. Munyinyi. 1995. Biocontrol potencial of entomogenous fungi Beauveria bassiana and Metarhizium anisopliae for tsé-tsé flies (Glossina spp.) of development sites. Journal of Invertebrate Pathology, 66: 237-241.

Lecuona, R., B. Papierok \& G. Riba. 1996. Hongos entomopatógenos. Pp. 35-60. In: R. E. Lecuona (Ed). Microorganismos patógenos empleados en el control microbiano de insectos plaga. Buenos Aires, Argentina.

Lezama-Gutiérrez, R., A. Trujillo-de la Luz, J. Molina-Ochoa, O. Rebolledo-Domínguez, A. R. Pescador, M. López-Edwards \& M. Aluja. 2000. Virulence of Metarhizium anisopliae (Deuteromycotina: Hyphomycetes) on Anastrepha ludens (Diptera: Tephritidae): Laboratory and field trials. Journal of Economic Entomology, 93: 1080-1084.

Little, T. M. \& H. Jackson. 1989. Métodos estadísticos para la investigación en la agricultura. Edit. Trillas. México, D.F.

Maniania, N. K. \& A. Ondulaja. 1998. Effect of species, age, and sex of tse-tse on response to infection by Metarhizium anisopliae. Biocontrol, 43: 311-323.

Samuels, R. I. \& I. C. Paterson. 1995. Cuticle degrading proteases from insect moulting fluid and culture filtrates of entomopathogenic fungus. Biochemistry and Molecular Biology, 110: 661-669. 
SAS Institute. 1992. SAS user's guide, versión 6.04. SAS Institute, Cary, NC.

Saul, S. H., D. Tsuda \& T. T. Y. Wong. 1983. Laboratory and field trials soil applications of methoprene and other insecticides for control of the Mediterranean fruit fly (Diptera: Tephritidae). Journal of Economic Entomology, 76: 174-177.

Penrose, D. 1993. The 1989/1990 Mediterranean fruit fly eradication program in California. Pp. 401406. In: M. Aluja and P. Liedo (Eds). Fruit flies: Biology and management. Springer-Verlag New York, Inc., USA.

Tanada, Y. 1963. Epizootiology fo infections disease. Pp. 423-475. In: E. A. Steinhaus (Ed). Insect Pathology. An advanced treatise. Vol. 2. Academic Press. New York, USA.

Toledo, J., S. E. Campos, S. Flores, P. Liedo, J. F. Barrera, A. Villaseñor \& P. Montoya. 2007. Horizontal transmission of Beauveria bassiana, Anastrepha ludens (Diptera: Tephritidae) under laboratory and field cage conditions. Journal of Economic Entomology, 100: 291-297.

Watson, D. W., C. J. Geden, S. J. Long \& D. A. Rutz. 1995. Efficacy of Beauveria bassiana for the controlling the house fly and stable fly (Diptera: Muscidae). Biological Control, 5: 405-411.

Watanabe, H. 1987. The host population. Pp. 71-112. In: J. R. Fuxa and Y. Tanada. (Eds). Epizootiology of insect diseases. John Wiley. New York, USA.

Villaseñor, A., J. Carrillo, J. Zavala, J. Stewart, C. Lira \& J. Reyes. 2000. Current progress in the medfly program Mexico-Guatemala. Pp. 361-368. In: K. H. Tan (Ed). Area-wide control of fruit flies and other insect pests. Penerbit Universiti Sains Malaysia. Penang, Malaysia.

Vargas, R.I., S. L. Peck, G. T. McQuate, C. G. Jackson, J. D. Stark \& J. W. Armstrong. 2001. Potential for area-wide integrated management of Mediterranean fruit fly (Diptera: Tephritidae) with a braconid parasitoid and a novel bait spray. Journal of Economic Entomology, 94: 817-825.

Yee, W. L. \& L. A. Lacey. 2005. Mortality of different life stages of Rhagoletis indifferens (Diptera: Tephritidae) exposed to the entomopathogenic fungus Metarhizium anisopliae. Journal of Entomological Science, 40: 167-177.

Zaragoza, A. \& L. Fernández. 2002. Biolure (3-component lure) mass trapping in citrus. Pp. 80. In: Abstracts of the $6^{\text {th }}$ International Symposium of Fruit Flies of Economic Importance. Stellenbosch, South Africa. 